\title{
Fuzzy Granularity in the Knowledge-based Dynamic Fuzzy Sets
}

\author{
Rolly Intan \\ Petra Christian University \\ Siwalankerto 121-131, Wonocolo \\ Surabaya 60236, Indonesia \\ +62-31-8439040 \\ rintan@petra.ac.id
}

\author{
Siana Halim \\ Petra Christian University \\ Siwalankerto 121-131, Wonocolo \\ Surabaya 60236, Indonesia \\ +62-31-8439040 \\ halim@petra.ac.id
}

\author{
Lily Puspa Dewi \\ Petra Christian University \\ Siwalankerto 121-131, Wonocolo \\ Surabaya 60236, Indonesia \\ $+62-31-8439040$ \\ lily@petra.ac.id
}

\begin{abstract}
In 2002, Intan and Mukaidono proposed Knowledge-based Fuzzy Sets (KFS) as an extended concept of the fuzzy set. Here, the membership function of a fuzzy set is subjectively determined by the knowledge. Wang et al. (1988) generalized the concept of fuzzy set, called Dynamic Fuzzy Sets (DFS). In the DFS, the membership degree of an element might dynamically change according to the time's variable. Both extended concepts of fuzzy sets were then combined by Intan et al. to be a hybrid concept, called Knowledgebased Dynamic Fuzzy Set. The concept is regarded as a more generalization of fuzzy sets by considering that the membership function of a given fuzzy set provided by a certain knowledge may be dynamically changed over time as usually happened in the realworld application. To continually extend the concept of knowledge-based dynamic fuzzy sets, this paper discusses how the fuzzy granularity is constructed in the knowledge-based dynamic fuzzy sets. The concepts of objectivity and consistency are discussed, along with their proposed measures.
\end{abstract}

\section{CCS Concepts}

$\mathrm{CCS} \rightarrow$ Computing methodologies $\rightarrow$ Artificial intelligence $\rightarrow$ Knowledge representation and reasoning $\rightarrow$ Vagueness and fuzzy logic

\section{Keywords}

Knowledge-based Dynamic Fuzzy Sets; Fuzzy Granularity; Fuzzy Coverings; Objectivity Measure; Consistency Measure.

\section{INTRODUCTION}

L.A. Zadeh introduced the concept of fuzzy set in $1965[1,2]$ as a generalization of crisp sets regarding the membership degree of elements. Here, the membership degrees of elements in a fuzzy set is given gradually by a real number started from 0 (non-member) to 1 (member).

In the concept of fuzzy sets proposed by L.A. Zadeh, the membership degree of an element given by a membership function is unchangeable regarding the time variable. However, it is well known that in the real-world application, everything is always changing dealing with time. Thus, the membership degree of an element is also possibly changeable anytime. To present this reality, Wang et al. (1988) [3, 4] introduced Dynamic Fuzzy Sets (DFS). The DFS might be considered as an extended concept of fuzzy sets, for each membership degree of an element in DFS is dynamically changeable dealing with time's variable. Here, the DFS may also be considered as multi-fuzzy sets by means that every time a given fuzzy label may have different fuzzy sets represented by different membership functions dealing with time variable.

As discussed by Intan and Mukaidono (2002) [5, 6, 7], the probability is a concept to solve the problem of objective uncertainty. On the other hands, fuzziness is regarded as a concept to represent a subjective uncertainty. A certain knowledge subjectively determines the membership degree of an element in a given fuzzy set. To realize this situation, Intan and Mukaidono (2002) $[5,6,7]$ introduced an extended concept of fuzzy sets, called Knowledge-based Fuzzy Sets (KFS). Like the DFS, the KFS may also be considered as multi-fuzzy sets by means that every fuzzy label may have different fuzzy sets represented by different pieces of knowledge. Here, we may consider fuzziness as a deterministic uncertainty by means that even in an uncertain (unclear) situation or definition of a given object, a person through his/ her knowledge may be subjectively able to determine the object. Therefore, a given fuzzy label may have $\mathrm{n}$ different membership functions (fuzzy sets) based on $\mathrm{n}$ different pieces of knowledge.

Since DFS and KFS are two extended concepts of fuzzy sets with different interpretations, it is possible to combine them to provide a more general concept. In this case, a membership function of a fuzzy set given by a specific knowledge is possibly changeable over time. Therefore, both extended concepts of fuzzy sets were then combined by Intan et al. [6] to be a hybrid concept, called Knowledge-based Dynamic Fuzzy Set (KDFS). The concept is regarded as a more generalization of fuzzy sets by considering that the membership function of a given fuzzy set provided by a specific knowledge may be dynamically changed over time as usually happened in the real-world application. We may consider the KDFS as a concept of two-dimensional multi-fuzzy sets dealing with time and knowledge. Three kinds of summary fuzzy sets provided by the aggregation functions were proposed and discussed. Also, some basic operations and properties of KDFS such as equality, contentment, union, intersection and complement were defined and examined.

This paper discusses how the fuzzy granularity, e.g. the crisp and fuzzy coverings of pieces of knowledge is constructed in the knowledge-based dynamic fuzzy sets. Crisp and fuzzy similarity classes of pieces of knowledge are created based on the conditional probability relations. The concepts of objectivity and consistency are discussed, along with their proposed measures.

\section{KNOWLEDGE-BASED DYNAMIC FUZZY SETS (KDFS)}

Intan et al. [8] introduced an extended concept of fuzzy sets, called Knowledge-based Dynamic Fuzzy Sets as a hybrid concept between dynamic fuzzy sets and knowledge-based fuzzy sets. The 
concept presented the practical world application that a particular membership function of fuzzy set $A$ given by a specific knowledge $k$ may dynamically changeable over the time. The following definition formally defines knowledge-based dynamic fuzzy sets.

Definition 1 Let $U$ be a universal set of elements, $K$ be a set of pieces of knowledge and $T \subseteq R^{+}$be a set of time, where $R^{+}=$ $[0, \infty)$. Then a knowledge-based dynamic fuzzy set $A$ on $U$ based on the knowledge $k \in K$, denoted by $A_{k}$, is defined and characterized by the following membership function.

$$
A_{k}: T \times U \rightarrow[0,1]
$$

Related to (1), $A_{k}(t, u) \in[0,1]$ is the membership degree of element $u \in U$ in fuzzy set $A$ based on knowledge $k \in K$ at the time $t \in T$. Similarly, $A_{k}(t, u)=1$ means $u$ is a full member of $A$ based on $k$ at the time $t$. On the other hand, $A_{k}(t, u)=0$ means $u \in U$ is not a member of $A_{k}(t)$. Thus, the membership degree of an element $u$ in $A$ may also vary depending on both the knowledge $k$ and the time $t$. Here, $A_{k}(t) \in \mathcal{F}(U)$ is regarded as a knowledgebased dynamic fuzzy set of $A$ which is based on knowledge $k$ at the time $t . A_{k}(t)$ is also a similar concept to the fuzzy set defined by Zadeh in $1965[7,8]$, where $\mathcal{F}(U)$ is a fuzzy power set of $U$. Set of knowledge-based dynamic fuzzy sets $A$ on $U$, denoted by $\mathcal{D}(A)$ is given by $\mathcal{D}(A)=\left\{A_{k}(t) \mid k \in K, t \in T\right\}$.

The relation among dynamic fuzzy sets, knowledge-based fuzzy sets and knowledge-based dynamic fuzzy sets was discussed by Intan et al. in [8]. Also, fuzzy summary sets, namely the time-based summary fuzzy set, the knowledge-based summary fuzzy set and general summary fuzzy set were proposed and discussed in detail using some proposed aggregate functions. Some basic operations and properties in the relation to the summary fuzzy sets are discussed and examined.

\subsection{Basic Operations and Properties}

As defined and proposed in [8], some basic operations of the knowledge-based dynamic fuzzy sets were discussed and defined as follows.

Definition 2 Let $U$ be a universal set of elements, $K$ be a set of pieces of knowledge and $T \subseteq R^{+}$be a set of time, where $R^{+}=$ $[0, \infty) . A$ and $B$ are two fuzzy sets on $U$. The following equations give some basic operations and properties of Equality, Containment, Union, Intersection and Complementation.

Equality

1. $A_{k}(t)=B_{k}(t) \Leftrightarrow A_{k}(t, u)=B_{k}(t, u), \forall u \in U$,

2. $A=B \Leftrightarrow A_{k}(t, u)=B_{k}(t, u), \forall u \in U, \forall k \in K, \forall t \in T$,

3. $A \cong B \Leftrightarrow A_{k}\left(t_{j_{1}}, u\right)=B_{k}\left(t_{j_{2}}, u\right)$,

$\forall u \in U, \forall k \in K, \forall t_{j_{1}}, t_{j_{2}} \in T$,

4. $A \triangleq B \Leftrightarrow A_{k_{i_{1}}}(t, u)=B_{k_{i_{2}}}(t, u)$, $\forall u \in U, \forall k_{i_{1}}, k_{i_{2}} \in K, \forall t \in T$,

5. $A \equiv B \Leftrightarrow A_{k_{i_{1}}}\left(t_{j_{1}}, u\right)=B_{k_{i_{2}}}\left(t_{j_{2}}, u\right)$, $\forall u \in U, \forall k_{i_{1}}, k_{i_{2}} \in K, \forall t_{j_{1}}, t_{j_{2}} \in T$,

6. $k_{i_{1}}=k_{i_{2}} \Leftrightarrow A_{k_{i_{1}}}(t, u)=A_{k_{i_{2}}}(t, u), \forall u \in U, \forall t \in T$, $\forall A \in \mathcal{F}(U)$, where $\mathcal{F}(U)$ is fuzzy power set on $U$.

\section{Containment}

7. $A_{k}(t) \subseteq B_{k}(t) \Leftrightarrow A_{k}(t, u) \leq B_{k}(t, u), \forall u \in U$,

8. $A \subseteq B \Leftrightarrow A_{k}(t, u) \leq B_{k}(t, u), \forall u \in U, \forall k \in K, \forall t \in T$,

9. $A \sqsubseteq B \Leftrightarrow A_{k}\left(t_{j_{1}}, u\right) \leq B_{k}\left(t_{j_{2}}, u\right)$,

$\forall u \in U, \forall k \in K, \forall t_{j_{1}}, t_{j_{2}} \in T$,
10. $A \preccurlyeq B \Leftrightarrow A_{k_{i_{1}}}(t, u) \leq B_{k_{i_{2}}}(t, u)$,

$\forall u \in U, \forall k_{i_{1}}, k_{i_{2}} \in K, \forall t \in T$,

11. $A \subseteq B \Leftrightarrow A_{k_{i_{1}}}\left(t_{j_{1}}, u\right) \leq B_{k_{i_{2}}}\left(t_{j_{2}}, u\right)$,

$\forall u \in U, \forall k_{i_{1}}, k_{i_{2}} \in K, \forall t_{j_{1}}, t_{j_{2}} \in T$,

12. $k_{i_{1}} \unlhd k_{i_{2}} \Leftrightarrow A_{k_{i_{1}}}(t, u) \leq A_{k_{i_{2}}}(t, u), \forall u \in U, \forall t \in T$,

$\forall A \in \mathcal{F}(U)$, where $\mathcal{F}(U)$ is fuzzy power set on $U$.

Union

13. $(A \cup B)_{k}(t, u)=\max \left(A_{k}(t, u), B_{k}(t, u)\right), \forall u \in U$,

14. $\left(A_{k_{i_{1}}}\left(t_{j_{1}}\right) \cup B_{k_{i_{2}}}\left(t_{j_{2}}\right)\right)(u)=\max \left(A_{k_{i_{1}}}\left(t_{j_{1}}, u\right), B_{k_{i_{2}}}\left(t_{j_{2}}, u\right)\right)$,

$\forall u \in U, t_{j_{1}}, t_{j_{2}} \in T, k_{i_{1}}, k_{i_{2}} \in K$,

Intersection

15. $(A \cap B)_{k}(t, u)=\min \left(A_{k}(t, u), B_{k}(t, u)\right), \forall u \in U$,

16. $\left(A_{k_{i_{1}}}\left(t_{j_{1}}\right) \cap B_{k_{i_{2}}}\left(t_{j_{2}}\right)\right)(u)=\min \left(A_{k_{i_{1}}}\left(t_{j_{1}}, u\right), B_{k_{i_{2}}}\left(t_{j_{2}}, u\right)\right)$,

$\forall u \in U, t_{j_{1}}, t_{j_{2}} \in T, k_{i_{1}}, k_{i_{2}} \in K$,

Complementation

17. $\neg A_{k_{i}}\left(t_{j}, u\right)=1-A_{k_{i}}\left(t_{j}, u\right)$,

18. $A_{\neg k_{i}}\left(t_{j}, u\right)=$

$$
\left\{\begin{array}{c}
A_{k_{r}}\left(t_{j}, u\right), r \neq i,|K|=2, \\
\Theta\left(\alpha_{k_{1}}, \cdots, \alpha_{k_{i-1}}, \alpha_{k_{i+1}}, \cdots, \alpha_{k_{n}}\right),|K|>2, \alpha_{k_{p}}=A_{k_{p}}\left(t_{j}, u\right),
\end{array}\right.
$$

19. $A_{k_{i}}\left(\neg t_{j}, u\right)=$

$$
\left\{\begin{array}{c}
A_{k_{i}}\left(t_{r}, u\right), r \neq j,|T|=2, \\
\Upsilon\left(\beta_{t_{1}}, \cdots, \beta_{t_{j-1}}, \beta_{t_{j+1}}, \cdots, \beta_{t_{m}}\right),|T|>2, \beta_{t_{p}}=A_{k_{i}}\left(t_{p}, u\right),
\end{array}\right.
$$

More basic operations and properties related to the summary fuzzy sets could be found in [3].

\section{GRANULARITY OF KNOWLEDGE}

As discussed by Intan and Mukaidono $[5,6,7]$ in proposing the concept of knowledge-based fuzzy sets, the granularity of knowledge was constructed to obtain the similarity classes of knowledge. All knowledge in a specific similarity class will consider having a similar perception subjectively toward a given fuzzy set. Through the similarity classes of knowledge, this paper discusses and introduces three necessary measures, namely Objectivity Measures, Individuality Measures and Consistency Measure in the knowledge-based dynamic fuzzy sets. Here, the similarity classes of knowledge are provided by a fuzzy conditional probability relation $[5,6,7]$ which is an asymmetric relation as defined by Definition 3 .

Definition 3 A fuzzy conditional probability relation is a mapping, $R: \mathcal{F}(U) \times \mathcal{F}(U) \rightarrow[0,1]$ such that for $X, Y \in \mathcal{F}(U)$,

$$
R(X, Y)=\frac{\sum_{u \in U} \min (X(u), Y(u))}{\sum_{u \in U} Y(u)}
$$

where $R(X, Y)$ means the degree $Y$ supports $X$ or the degree $Y$ is similar to $X$ or similarity degree of $X$ given $Y$.

An interesting mathematical relation characterizes the concept of fuzzy conditional probability relation. This relation is called weak fuzzy similarity relationship and defined as follows.

Definition 4 A weak fuzzy similarity relation is a mapping, $S: \mathcal{F}(U) \times \mathcal{F}(U) \rightarrow[0,1]$, such that for $X, Y, Z \in \mathcal{F}(U)$,

1. Reflexivity: $S(X, X)=1$

2. Conditional symmetry: if $S(X, Y)>0$ then $S(Y, X)>0$

3. Conditional transitivity: If $S(X, Y) \geq S(Y, X)>0$ and $S(Y, Z) \geq S(Z, Y)>0$ then 


$$
S(X, Z) \geq S(Z, X)>0
$$

where $U$ is an ordinary set of elements and $\mathcal{F}(U)$ is fuzzy power sets of $U$.

Furthermore, in the relation to (2), similarity degree of $k_{i_{1}}$ given $k_{i_{2}}$ concerning fuzzy set $A$ in time $t$ is given by the following equation.

$R\left(A_{k_{i_{1}}}(t), A_{k_{i_{2}}}(t)\right)=\frac{\sum_{u \in U} \min \left(A_{k_{i_{1}}}(t, u), A_{k_{i_{2}}}(t, u)\right)}{\sum_{u \in U} A_{k_{i_{2}}}(t, u)}$

It can be followed clearly that the degree of similarity between two knowledge satisfy the following properties.

$$
\begin{aligned}
\text { r1. } & {\left[R\left(A_{k_{i_{1}}}(t), A_{k_{i_{2}}}(t)\right)=R\left(A_{k_{i_{2}}}(t), A_{k_{i_{1}}}(t)\right)=1, \forall A \in\right.} \\
& \mathcal{F}(U), \forall t \in T] \Leftrightarrow k_{i_{1}}=k_{i_{2}} \\
\text { r2. } & {\left[R\left(A_{k_{i_{2}}}(t), A_{k_{i_{1}}}(t)\right)=1, R\left(A_{k_{i_{1}}}(t), A_{k_{i_{2}}}(t)\right)<1, \forall A \in\right.} \\
& \mathcal{F}(U), \forall t \in T] \Leftrightarrow k_{i_{1}} \unlhd k_{i_{2}} \\
\text { r3. } & {\left[R\left(A_{k_{i_{2}}}(t), A_{k_{i_{1}}}(t)\right)=R\left(A_{k_{i_{1}}}(t), A_{k_{i_{2}}}(t)\right)>0, \forall A \in\right.} \\
& \mathcal{F}(U), \forall t \in T] \Leftrightarrow k_{i_{1}} \sim k_{i_{2}} \\
\text { r4. } & {\left[R\left(A_{k_{i_{1}}}(t), A_{k_{i_{2}}}(t)\right)<R\left(A_{k_{i_{2}}}(t), A_{k_{i_{1}}}(t)\right), \forall A \in \mathcal{F}(U), \forall t \in\right.} \\
& T] \Leftrightarrow k_{i_{1}} \preccurlyeq k_{i_{2}} \\
\text { r5. } & R\left(A_{k}(t), A_{k}(t)\right)=1, \forall t \in T, \forall k \in K, \forall A \in \mathcal{F}(U) \\
\text { r6. } & {\left[R\left(A_{k_{i_{1}}}(t), A_{k_{i_{2}}}(t)\right)>0, \forall A \in \mathcal{F}(U), \forall t \in T\right] \Leftrightarrow } \\
& {\left[R\left(A_{k_{i_{2}}}(t), A_{k_{i_{1}}}(t)\right)>0\right] } \\
\text { r7. }[ & {\left[R\left(A_{k_{i_{1}}}(t), A_{k_{i_{2}}}(t)\right) \geq R\left(A_{k_{i_{2}}}(t), A_{k_{i_{1}}}(t)\right),\right.} \\
& R\left(A_{k_{i_{2}}}(t), A_{k_{i_{3}}}(t)\right) \geq R\left(A_{k_{i_{3}}}(t), A_{k_{i_{2}}}(t)\right), \forall A \in \mathcal{F}(U), \forall t \in \\
& T] \Rightarrow\left[R\left(A_{k_{i_{1}}}(t), A_{k_{i_{3}}}(t)\right) \geq R\left(A_{k_{i_{3}}}(t), A_{k_{i_{1}}}(t)\right)\right]
\end{aligned}
$$

Property (r1) is to prove that both knowledge, $k_{i_{1}}$ and $k_{i_{2}}$ are the same, and it is similar to Equality (6). (r2) shows that $k_{i_{2}}$ covers $k_{i_{1}}$, or $k_{i_{1}}$ contains in $k_{i_{2}}$. It means that in all the time, $k_{i_{2}}$ gives a higher degree of membership for all element of all fuzzy sets than $k_{i_{1}}$, and it is the same as Containment (12). Property (r3) points to similar cardinality between $k_{i_{1}}$ and $k_{i_{2}}$ for all fuzzy sets in all the time. On the other hand, (r4) means the cardinality of all fuzzy sets and all the time is given by $k_{i_{1}}$ is always less or equal to $k_{i_{2}}$. As related to the weak fuzzy similarity relation, $(\mathrm{r} 5)$ is the property of reflexivity. (r6) is a conditional similarity, and (r7) is a conditional transitivity.

Using degree of similarity between two pieces of knowledge as calculated by (3), two asymmetric similarity classes of a given element of knowledge $k$.

Definition 4 Let $K$ be a non-empty universal set of knowledge, and $A$ be a fuzzy set on $U$. For any $k_{i} \in K, S_{\alpha}^{A}\left(k_{i}, t\right)$ and $P_{\alpha}^{A}\left(k_{i}, t\right)$ are defined as the set of knowledge that supports $k_{i}$ and the set supported by $k_{i}$ at time $t \in T$, respectively by:

$$
\begin{aligned}
& S_{\alpha}^{A}\left(k_{i}, t\right)=\left\{k \in K \mid R\left(A_{k_{i}}(t), A_{k}(t)\right)>\alpha\right\} \\
& P_{\alpha}^{A}\left(k_{i}, t\right)=\left\{k \in K \mid R\left(A_{k}(t), A_{k_{i}}(t)\right)>\alpha\right\}
\end{aligned}
$$

where $\alpha \in[0,1]$.

$S_{\alpha}^{A}\left(k_{i}, t\right)$ can also be interpreted as the set of knowledge that is similar to $k_{i}$ at time $t$ with respect to fuzzy set $A$. On the other hand, $P_{\alpha}^{A}\left(k_{i}, t\right)$ can be considered as the set of knowledge to which $k_{i}$ is similar. In this case, $S_{\alpha}^{A}\left(k_{i}, t\right)$ and $P_{\alpha}^{A}\left(k_{i}, t\right)$ are regarded as two different semantic interpretations of similarity classes in providing the crisp granularity of knowledge.

For two asymmetric similarity classes of knowledge, $S_{\alpha}^{A}\left(k_{i_{1}}, t\right)$ and $S_{\alpha}^{A}\left(k_{i_{2}}, t\right)$, the complement, intersection and union are defined by:

$$
\begin{gathered}
\neg S_{\alpha}^{A}\left(k_{i_{1}}, t\right)=\left\{k \in K \mid k \notin S_{\alpha}^{A}\left(k_{i_{1}}, t\right)\right\} \\
S_{\alpha}^{A}\left(k_{i_{1}}, t\right) \cap S_{\alpha}^{A}\left(k_{i_{2}}, t\right)= \\
\left\{k \in K \mid k \in S_{\alpha}^{A}\left(k_{i_{1}}, t\right) \text { and } k \in S_{\alpha}^{A}\left(k_{i_{2}}, t\right)\right\} \\
S_{\alpha}^{A}\left(k_{i_{1}}, t\right) \cup S_{\alpha}^{A}\left(k_{i_{2}}, t\right)= \\
\left\{k \in K \mid k \in S_{\alpha}^{A}\left(k_{i_{1}}, t\right) \text { or } k \in S_{\alpha}^{A}\left(k_{i_{2}}, t\right)\right\}
\end{gathered}
$$

Similarly, the complement, intersection and union might be defined on $P_{\alpha}^{A}\left(k_{i_{1}}, t\right)$ and $P_{\alpha}^{A}\left(k_{i_{2}}, t\right)$. Since the similarity classes of knowledge are crisp sets, they satisfy the Boolean Lattice. Based on these two asymmetric similarity classes, we then construct two dynamic crisps covering of the universal knowledge regarding fuzzy set $A, \quad \Upsilon_{A}^{\alpha}(t)=\left\{P_{\alpha}^{A}(k, t) \mid k \in K\right\}$ and $\Psi_{A}^{\alpha}(t)=$ $\left\{S_{\alpha}^{A}(k, t) \mid k \in K\right\}$, where $\alpha \in[0,1]$. Here the crisp, dynamic covering means that the crisp covering will be dynamically changed depending on time $t$.

By removing $\alpha$, crisp similarity classes, $S_{\alpha}^{A}\left(k_{i}, t\right)$ and $P_{A}^{\alpha}\left(k_{i}, t\right)$ will be generalized to the fuzzy similarity classes, $S_{k_{i}}^{A}(t)$ and $P_{k_{i}}^{A}(t)$, respectively. Naturally, the fuzzy similarity classes of a specific knowledge $k_{i}$ with respect to fuzzy set $A$ at time $t$ is given by the following equations.

$$
\begin{gathered}
S_{k_{i}}^{A}(t, k)=R\left(A_{k_{i}}(t), A_{k}(t)\right), \forall k \in K \\
P_{k_{i}}^{A}(t, k)=R\left(A_{k}(t), A_{k_{i}}(t)\right), \forall k \in K
\end{gathered}
$$

Basic operations, such as the complement, intersection and union of the fuzzy similarity classes are defined by:

$$
\begin{aligned}
& \neg S_{k_{i}}^{A}(t, k)=1-S_{k_{i}}^{A}(t, k), \forall k \in K \\
& S_{k_{i_{1}}}^{A}(t, k) \wedge S_{k_{i_{2}}}^{A}(t, k)=\min \left(S_{k_{i_{1}}}^{A}(t, k), S_{k_{i_{2}}}^{A}(t, k)\right), \forall k \in K \\
& S_{k_{i_{1}}}^{A}(t, k) \vee S_{k_{i_{2}}}^{A}(t, k)=\max \left(S_{k_{i_{1}}}^{A}(t, k), S_{k_{i_{2}}}^{A}(t, k)\right), \forall k \in K
\end{aligned}
$$

Furthermore, two dynamic fuzzy coverings of the universal set of knowledge are constructed dealing with a fuzzy set $A$ as defined by $\Theta^{A}(t)=\left\{P_{k}^{A}(t) \mid k \in K\right\}$ and $\Omega^{A}(t)=\left\{S_{k}^{A}(t) \mid k \in K\right\}$. Here, the fuzzy coverings of the universal set of knowledge are also dynamically changed based on the time $t$.

\section{OBJECTIVITY AND CONSISTENCY MEASURES}

Concerning the knowledge-based dynamic fuzzy sets, it is necessary to discuss and propose objectivity, individuality and consistency measures. In general, a given object is described objectively if and only if its description can be accepted by all persons (knowledge). Naturally, it may say that the more a given description is acceptable or agreeable means the description is more objective. Since in the knowledge-based dynamic fuzzy sets, a given fuzzy label may have many membership functions provided by knowledge, it is necessary to propose a measure, called objectivity measure, to calculate the degree of objectivity regarding the knowledge-based dynamic fuzzy sets. First, objectivity measure is defined on the dynamic crisp covering of knowledge as follows.

Definition 5 Let $K$ be a non-empty universal set of knowledge, and $P_{\alpha}^{A}\left(k_{i}, t\right)$ is a set of knowledge that is supported by $k_{i} . \varphi_{\alpha}^{A}\left(k_{i}, t\right)$ is defined as a function to calculate the degree of objectivity $k_{i}$ in 
dealing fuzzy label $A$ at time $t$ in the degree of similarity greater than $\alpha$ by:

$$
\varphi_{\alpha}^{A}\left(k_{i}, t\right)=\frac{\left|P_{\alpha}^{A}\left(k_{i}, t\right)\right|}{|K|}
$$

where $\alpha \in[0,1]$.

Instead of using $S_{\alpha}^{A}\left(k_{i_{1}}, t\right),(14)$ uses $P_{\alpha}^{A}\left(k_{i}, t\right)$ in calculating the degree of objectivity because intuitively we would like to find the similarity of others given $k_{i_{1}}$. As a contrary to the objectivity measure, individuality measure may be defined as follows.

Definition 6 Let $K$ be a non-empty universal set of knowledge, and $P_{\alpha}^{A}\left(k_{i}, t\right)$ is a set of knowledge that is supported by $k_{i} . \vartheta_{\alpha}^{A}\left(k_{i}, t\right)$ is defined as a function to calculate the degree of individuality $k_{i}$ in dealing fuzzy label $A$ at time $t$ in the degree of similarity greater than $\alpha$ by:

$$
\vartheta_{\alpha}^{A}\left(k_{i}, t\right)=\frac{\left|K-P_{\alpha}^{A}\left(k_{i}, t\right)\right|+1}{|K|},
$$

where $\alpha \in[0,1]$.

Relation between $\varphi_{\alpha}^{A}\left(k_{i}, t\right)$ and $\vartheta_{\alpha}^{A}\left(k_{i}, t\right)$ is given by the following equations.

$$
\begin{aligned}
& \text { (a) } \varphi_{\alpha}^{A}\left(k_{i}, t\right), \vartheta_{\alpha}^{A}\left(k_{i}, t\right) \in\left\{\frac{1}{|K|}, \frac{2}{|K|}, \cdots, 1\right\}, \\
& \text { (b) } \varphi_{\alpha}^{A}\left(k_{i}, t\right)=1-\vartheta_{\alpha}^{A}\left(k_{i}, t\right)+\frac{1}{|K|} .
\end{aligned}
$$

From (a), it shows that the minimum degree of both $\varphi_{\alpha}^{A}\left(k_{i}, t\right)$ and $\vartheta_{\alpha}^{A}\left(k_{i}, t\right)$ equal to $\frac{1}{|K|}$. The minimum of objectivity cannot be 0 because when nobody supports or agrees with $k_{i}$ in its description to a fuzzy set $A$ at time $t$, at least there is one knowledge will support that is $k_{i}$ itself. However, even if all knowledge in $K$ support or agree with $k_{i}$, it does not mean that individuality of becoming extinct. Thus, similarly, the minimum degree of individuality also equals to $\frac{1}{|K|}$. Here, the degree of objectivity will be higher when more knowledge support and agree with $k_{i}$. On the other hand, the degree of individuality will be higher when less knowledge supports and agrees with $k_{i}$. Clearly, it can be proved that $\varphi_{\alpha}^{A}\left(k_{i}, t\right)=\vartheta_{\alpha}^{A}\left(k_{i}, t\right)=1 \Leftrightarrow|K|=1$. In addition, as given in Definition 5 and 6 , the degrees of objectivity and individuality rely on discrete value as a result of using crisp coverings. The most important thing is that both $\varphi_{\alpha}^{A}\left(k_{i}, t\right)$ and $\vartheta_{\alpha}^{A}\left(k_{i}, t\right)$ are dynamically changed over time $t$. It represents the real-world application that the objectivity of someone will always be changed over time. (b) show a simple equation representing the relation between $\varphi_{\alpha}^{A}\left(k_{i}, t\right)$ and $\vartheta_{\alpha}^{A}\left(k_{i}, t\right)$. It can be verified that $\varphi_{\alpha}^{A}\left(k_{i}, t\right)$ and $\vartheta_{\alpha}^{A}\left(k_{i}, t\right)$ satisfy some properties such as:

(a) $\varphi_{1}^{A}\left(k_{i}, t\right)=1, \vartheta_{1}^{A}\left(k_{i}, t\right)=\frac{1}{|K|} \Leftrightarrow k_{i} \unlhd k, \forall k \in K$

(b) $\varphi_{0}^{A}(k, t)=1, \vartheta_{0}^{A}(k, t)=\frac{1}{|K|}, \forall k \in K$

(c) $\alpha_{1} \leq \alpha_{2} \Leftrightarrow \varphi_{\alpha_{1}}^{A}(k, t) \geq \varphi_{\alpha_{2}}^{A}(k, t), \vartheta_{\alpha_{1}}^{A}(k, t) \vartheta_{\alpha_{2}}^{A}(k, t), \forall k \in K$

Consistency measure is represented by a function to calculate the degree of consistency regarding a specific knowledge $k$ in describing a given fuzzy set $A$ between two different times, $t_{j_{1}}$ and $t_{j_{2}}$. Formally, a consistency measure is defined in Definition 7.

Definition 7 Let $K$ and $T$ be two non-empty universal sets of knowledge and times, respectively. $A$ be a fuzzy set over $U$, where $U$ is a universal set of elements. $\eta_{k_{i}}^{A}\left(t_{j_{1}}, t_{j_{2}}\right)$ is defined as a function to calculate the degree of consistency regarding a certain knowledge $k_{i} \in K$ in describing a given fuzzy set $A$ between two different times, $t_{j_{1}} \in T$ and $t_{j_{2}} \in T$ as follows.

$$
\eta_{k_{i}}^{A}\left(t_{j_{1}}, t_{j_{2}}\right)=1-\frac{\sum_{u \in U}\left|A_{k_{i}}\left(t_{j_{1}}, u\right)-A_{k_{i}}\left(t_{j_{2}}, u\right)\right|}{|U|}
$$

From (16), it can be verified that $\eta_{k_{i}}^{A}\left(t_{j_{1}}, t_{j_{2}}\right)=1 \Leftrightarrow A_{k_{i}}\left(t_{j_{1}}\right)=$ $A_{k_{i}}\left(t_{j_{2}}\right), \forall k \in K, t_{j_{1}}, t_{j_{2}} \in T$. The bigger degree of consistency does not mean better or more accurate the description. It could be meant that the description is getting worse or more inaccurate. For instance, supposing that the object is changed from $t_{j_{1}}$ to $t_{j_{2}}$, the higher degree of consistency in describing the object between $t_{j_{1}}$ and $t_{j_{2}}$ may be more considered as inaccuracy. Therefore. It is necessary to also consider the degree of consistency in objectivity as given by Definition 8 .

Definition 8 Let $K$ and $T$ be two non-empty universal sets of knowledge and time, respectively. $A$ be a fuzzy set over $U$, where $U$ is a universal set of elements. $\omega_{\alpha}^{A}\left(k_{i}, t_{j_{1}}, t_{j_{2}}\right)$ is defined as a function to calculate the degree of consistency in objectivity regarding a certain knowledge $k_{i} \in K$ in describing a given fuzzy set $A$ between two different times, $t_{j_{1}} \in T$ and $t_{j_{2}} \in T\left(t_{j_{1}}<t_{j_{2}}\right)$ in the degree of similarity greater than $\alpha \in[0,1]$ by:

$$
\omega_{\alpha}^{A}\left(k_{i}, t_{j_{1}}, t_{j_{2}}\right)=\frac{\left|P_{\alpha}^{A}\left(k_{i}, t_{j_{1}}\right) \cap P_{\alpha}^{A}\left(k_{i}, t_{j_{2}}\right)\right|}{\left|P_{\alpha}^{A}\left(k_{i}, t_{j_{1}}\right)\right|}
$$

Obviously, it can be proved from (17) that $\omega_{\alpha}^{A}\left(k_{i}, t_{j_{1}}, t_{j_{2}}\right)=1 \Leftrightarrow$ $P_{\alpha}^{A}\left(k_{i}, t_{j_{1}}\right) \subseteq P_{\alpha}^{A}\left(k_{i}, t_{j_{2}}\right)$. Since $t_{j_{1}}<t_{j_{2}}$, this situation means the degree of objectivity in $t_{j_{1}}$ could be maintained or even higher in $t_{j_{2}}$. Thus, even degree of consistency between $t_{j_{1}}$ and $t_{j_{2}}$ $\left(\eta_{k_{i}}^{A}\left(t_{j_{1}}, t_{j_{2}}\right)\right)$ is low, high degree of consistency in objectivity $\left(\omega_{\alpha}^{A}\left(k_{i}, t_{j_{1}}, t_{j_{2}}\right)\right)$ shows that the accuracy is still maintained from $t_{j_{1}}$ to $t_{j_{2}}$.

\section{CONCLUSION}

This paper discussed how fuzzy granularity, especially crisp and fuzzy coverings of knowledge were constructed dealing with the concept of knowledge-based dynamic fuzzy sets. Crisp and fuzzy similarity classes of knowledge were created based on the conditional probability relations. The concepts of objectivity and consistency were discussed, along with their measurer to calculate the degree of objectivity and the degree of consistency. Their properties were examined.

\section{REFERENCES}

[1] Zadeh, L.A. 1990. Fuzzy Sets and systems. International Journal of General Systems 17, 129-138.

[2] Zadeh, L.A. 1965. Fuzzy Sets. Inf. Control 8, 338-353.

[3] Cai, M., Li, Q., Lang, G. 2017. Shadowed sets of dynamic fuzzy sets. Granular Computing 2, 85-94.

[4] Wang, G.Y., Ou, J.P., Wang, P.Z. 1988. Dynamic fuzzy sets. Fuzzy System Math 2(1), 1-8.

[5] Intan, R., Mukaidono, M., Emoto, M. 2002. Knowledgebased Representation of Fuzzy Sets. Proceeding of FUZZIEEE'02, 590-595.

[6] Intan, R., Mukaidono, M. 2002. Approximate Reasoning in Knowledge-based Fuzzy Sets. Proceeding of NAFIPSFLINT 2002, IEEE Publisher, 439-444.

[7] Intan, R., Mukaidono, M. 2002. On Knowledge-based Fuzzy Sets. International Journal of Fuzzy Systems 4(2), Chinese Fuzzy Systems Association T (CFSAT), 655-664.

[8] Intan, R., Halim, S., Dewi, L.P. 2018. On the Knowledgebased Dynamic Fuzzy Sets. Advances in Intelligent Systems and Computing. Springer. In Press. 


\section{Columns on Last Page Should Be Made As Close As Possible to Equal Length}

- The below form will not be published, but it will help us to understand your paper better

\section{Authors' background}

\begin{tabular}{|l|l|l|l|l|}
\hline Name & Email & $\begin{array}{l}\text { Position } \\
\text { (Prof, Assoc. Prof. } \\
\text { etc.) }\end{array}$ & Research Field & $\begin{array}{l}\text { Homepage } \\
\text { URL }\end{array}$ \\
\hline Rolly Intan & rintan@petra.ac.id & Professor & $\begin{array}{l}\text { Soft } \\
\text { Computing } \\
\text { (Fuzzy Sets, } \\
\text { Rough Sets, } \\
\text { Granular } \\
\text { Computing) }\end{array}$ & \\
\hline Siana Halim & halim@petra.ac.id & $\begin{array}{l}\text { Associate } \\
\text { Professor }\end{array}$ & $\begin{array}{l}\text { Statistical } \\
\text { Modeling }\end{array}$ & \\
\hline Lily Puspa Dewi & lily@petra.ac.id & $\begin{array}{l}\text { Senior } \\
\text { Lecturer }\end{array}$ & & \\
\hline & & & & \\
\hline
\end{tabular}

